\title{
A Suite of Mechanistic Epidemiological Decision Support Tools
}

\author{
Paul Fenimore*, Benjamin McMahon, Nicolas Hengartner, Timothy Germann and \\ Judith Mourant
}

Los Alamos National Laboratory, Los Alamos, NM, USA

\section{Objective}

We will demonstrate tools that allow mechanistic contraints on disease progression and epidemic spread to play off against interventions, mitigation, and control measures. The fundamental mechanisms of disease progression and epidemic spread provide important constraints on interpreting changing epidemic cases counts with time and geography in the context of on-going interventions, mitigations, and controls. Models such as these that account for the effects of human actions can also allow evaluation of the importance of categories of epidemic and disease controls.

\section{Introduction}

We present the EpiEarly, EpiGrid, and EpiCast tools for mechanistically-based biological decision support. The range of tools covers coarse-, medium-, and fine-grained models. The coarsegrained, aggregated time-series only data tool (EpiEarly) provides a statistic quantifying epidemic growth potential and associated uncertainties. The medium grained, geographically-resolved model (EpiGrid) is based on differential equation type simulations of disease and epidemic progression in the presence of various human interventions geared toward understanding the role of infection control, early vs. late diagnosis, vaccination, etc. in outbreak control. A fine-grained hybrid-agent epidemic model (EpiCast) with diurnal agent travel and contagion allows the analysis of the importance of contact-networks, travel, and detailed intervention strategies for the control of outbreaks and epidemics.

\section{Methods}

We use three types of methods for simulation and analysis. They are: (1) Bayesian and regression methods allowing estimation of the basic reproductive number from case-count data; (2) ordinarydifferential equation integration with modifications to account for discreteness of disease spread when case counts are small (we include space- and time-dependent effects); and (3) methods that hybridize agent-based travel, mixing, and disease progression with nested-mass action contagion (i.e. not fully agent-based). From the perspective of decision support, the crucial feature of mechanistic infectious epidemiological models is a way to capture the human interventions that determine epidemic outcome. Categorizing types of mitigation into those that change the force of infection, and those that branch disease progression allows a common framework that can be extended from medium-grained models through fine-grained. Our canonical example is our EpiGrid tool which allows for the modulation of the force of infection (i.e. contagion) with time (and potentially space), the vaccination of a susceptible population in a geographicallytargeted manner, movement controls, and branching our disease progression model to account for early- vs. late-intervention during host disease progression.

\section{Results}

We will present analysis of diseases that exemplify the various aspects of analysis in support of outbreak and epidemic control. Human and animal diseases relevant to this demonstration include rinderpest, avian influenza, and measles. We will begin with
EpiEarly's estimate of epidemic potential using aggregated timedependent case-count data. The key observation for EpiEarly is that under a wide range of situations a disease's reproductive number should be generalized to a distribution of possibilities to account for inherent randomness and other factors (including the variability of a disease contact network). We will then continue with a demonstration of EpiGrid's capabilities for understanding and modelling the role of interventions including contagion control (the force of infection), treatment (changing disease progression and infectiousness depending on treatment), vaccination, culling, and movement controls. We will briefly touch on the capabilities of EpiCast for more detailed analysis of specific intervention strategies.

\section{Conclusions}

We will demonstrate examples where modeling either contributed or plausibly would contribute to informing epidemic and outbreak control constrained by the possibilities of the underlying epidemic and disease dynamics.

\section{Keywords}

intervention; Geospatial; epidemic; disease progression

\section{Acknowledgments}

We thank the Defense Threat Reduction Agency for their support.

\section{*Paul Fenimore}

E-mail: paulf@lanl.gov 